\section{Investigating paedomorphism in the evolution of L. saxatilis}

Littorina saxatilis (rough periwinkle) is often used as different ecotypes adapted to life at different levels of the seashore. Professor Emilio Rolán-Alvarez and his colleagues at the University of Vigo, Spain investigate whether one ecotype may have evolved from another by paedomorphosis. while there is some thit, in support of paedomorphism in this species the direction of change in shell morphology across development is
not consistent with a paedomorphic hypothesis.

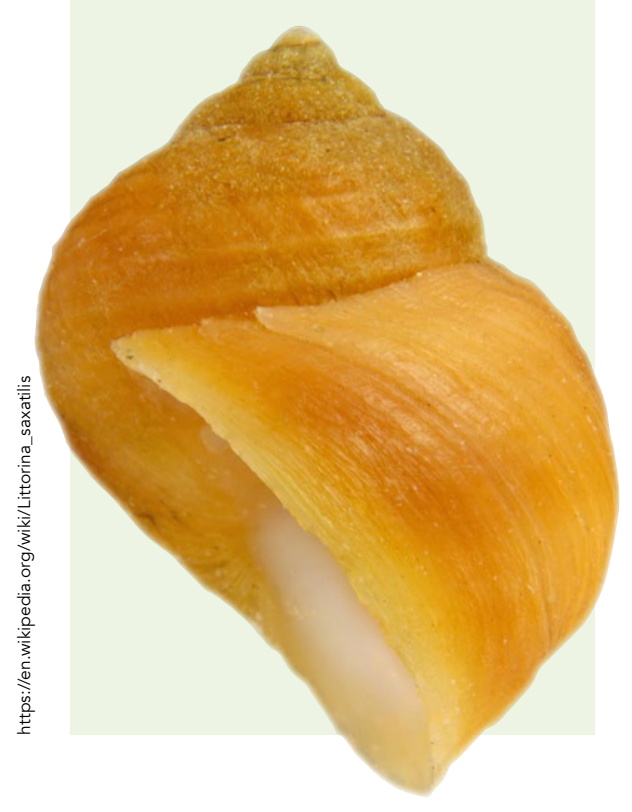

I ittorina saxatilis (L. saxatilis), $\quad$ organism. Influential paleontologist commonly known as rough periwinkle, Prof Stephen Jay Gould suggested that found on the intertidal rocky shores of (somatic cells being all cells other thanents used as an examples, germ cells, and stem cells) so to several ecotypes of $L$. saxatilis all that when the organism reaches maturity occupying different specific niches. Prof Emilio Rolán-Alvarez and his colleagues at the University of Vigo, Spain consider two ecotypes in particular, known as the "crab" and "wave" ecotypes. The wave ecotype lives on the lower intertidal shore, where it experiences constant waves an tides and is at risk of being dislodged. The adaptations to this niche include a smaller size, presumably to fit into gaps between rocks, and a larger shell apertur to acconmodate a large muscular foot crab ecotype lives higher on the shore, where it is atrik of dning out orbeing predated by crabs. A larger size, thicker shell, and a smaller shell aperture are the ideal adaptations to sunvive in this niche. There is some phylogenetic evidence that the ancestral form of these two ecotypes may have shared the crab ecotypes hypothesised that the wave ecotype could have evolved through paedomorphosis. Prof Rolán-Alvarez and his colleagues are investigating this possibility through proteomics and geometric

THE PAEDOMORPHIC HYPOTHESIS Paedomorphism is the retention of Juvenile characteristics in an adult

In L. saxatilis it has been suggested that the wave ecotype may have paedomorph crab ecotype throug that the wave ecotype has retained the small size and large shell aperture from a juvenile stage shared with the crab ecotype. There is an adaptive advantage of a smaller size for living on the lower shore, but the trade-off for a smaller size can be that this prevents reaching the required size/age for sexual maturation. Paedomorphism could be the solution for this problem. However, it can be very occurred in a species.

In a 2012 study, Prof Rolán-Alvarez and L. saxatils ecotypes. The proteome of a

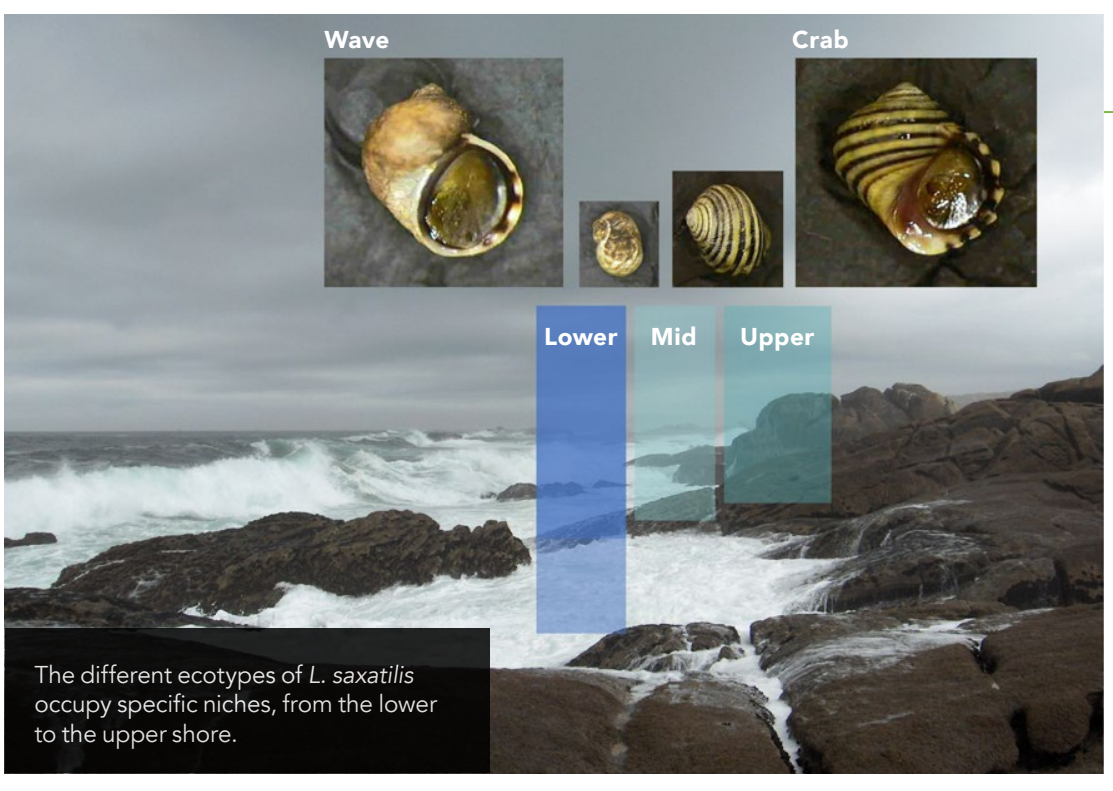

two ecotypes. They hypothesised tha the wave ecotype has evolved from the crab ecotype (or an ancestor with through paedomorphism, then the form of the wave ecotype shouldn't differentiate across development as much as the crab ecotype. Additionally, the adult form of the wave ecotype should bear a strong resemblance to the juvenile form of the crab ecotype.

or this experiment, samples of L. saxatilis were collected from the coast of northwest Spain and grouped into size classes (in this species size can be used as a proxy
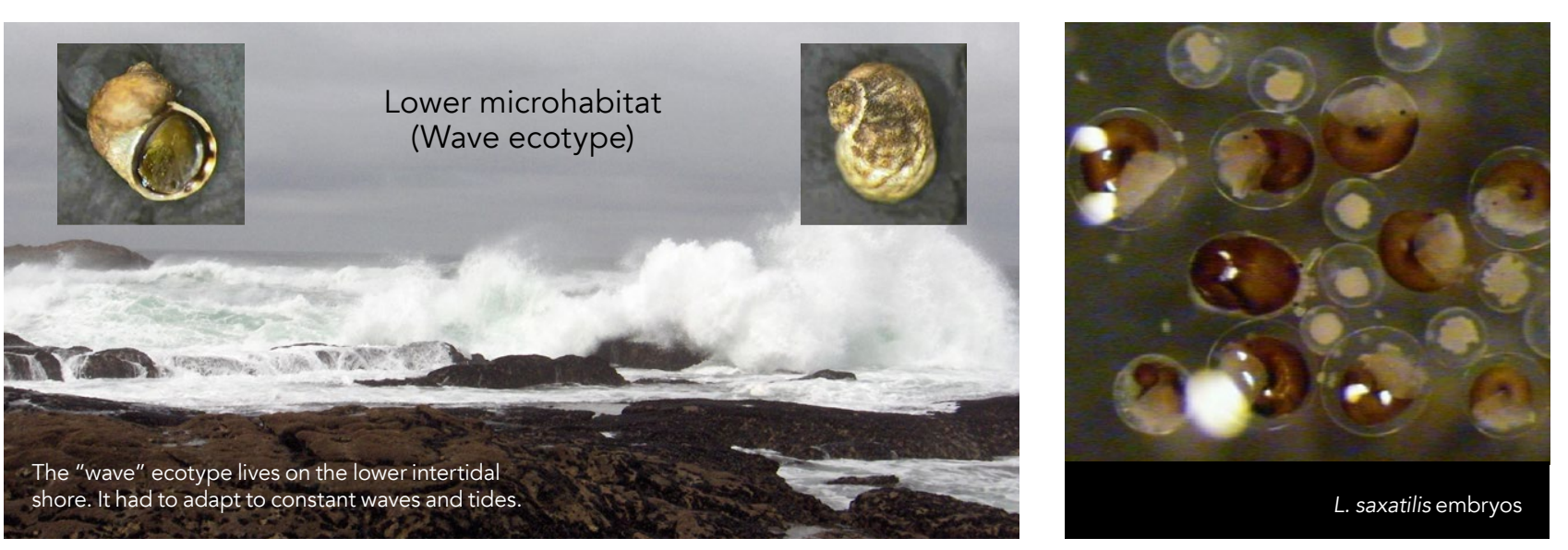

the proteomes of the two ecotypes level of proteomic a higher level of proteomic differentiation determined for each differentiation than differentiation than than the wave ecotype across ontogeny. based on the range the wave ecotype across ontogeny. to allow analysis of difficult to prove that paedomorphosis has colleagues analysed the proteome of the species is the complete set of proteins
This supports a paedomorphic hypothesis aduvenile characteristic retention in the proteomic differentiation. The analysis is limited though as it can only indicate that from an early stage of development, and cannot determine the exact changes that have taken place.

The differences between the crab and wave ecotypes of $L$. saxatilis are in the morphology of shell shape and size, and therefore a morphometric approach to testing the paedomorphic hypothesis could yield valuable results. In a 2020 paper, Prof Rolan-Alvarez and his collegues describe an experiment they conducted to investigate the changes in the ecotypes differ at the proteomic level

\section{GEOMETRIC MORPHOMETRICS}

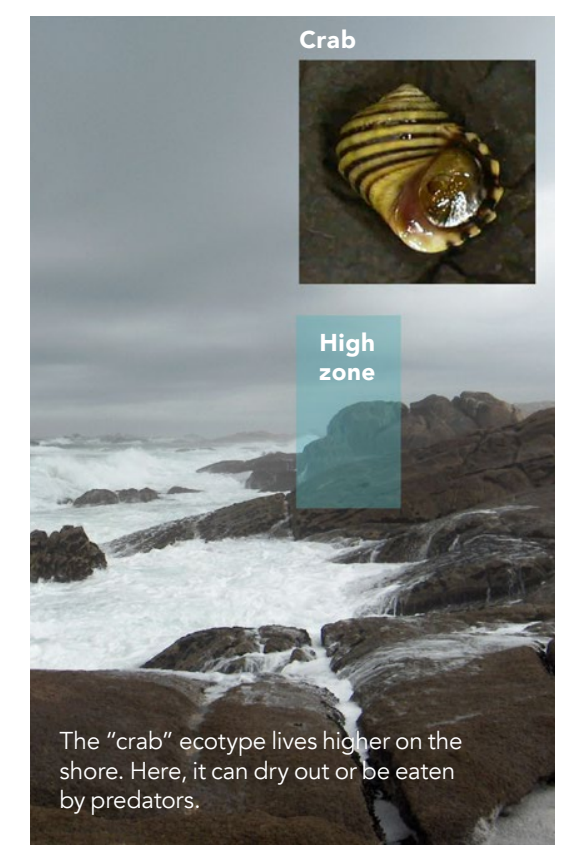

the change in shell size over time speric for that on the shells were used to establish overall size and the area of the shell aperture. The comparison of the change in shell sizes across the size classes showed that the crab ecotype changed more in terms of shell shape than the wave ecotype. These of proteomic differentiation found in the crab ecotype in the previous study, and support a paedomorphic hypothesis.

However, when the area of the shell aperture was considered across the size classes of the two ecotypes, it became clear that while the results might show the expected pattern of change, the parection of change doesn't support a of change refers to the magnitude of the 


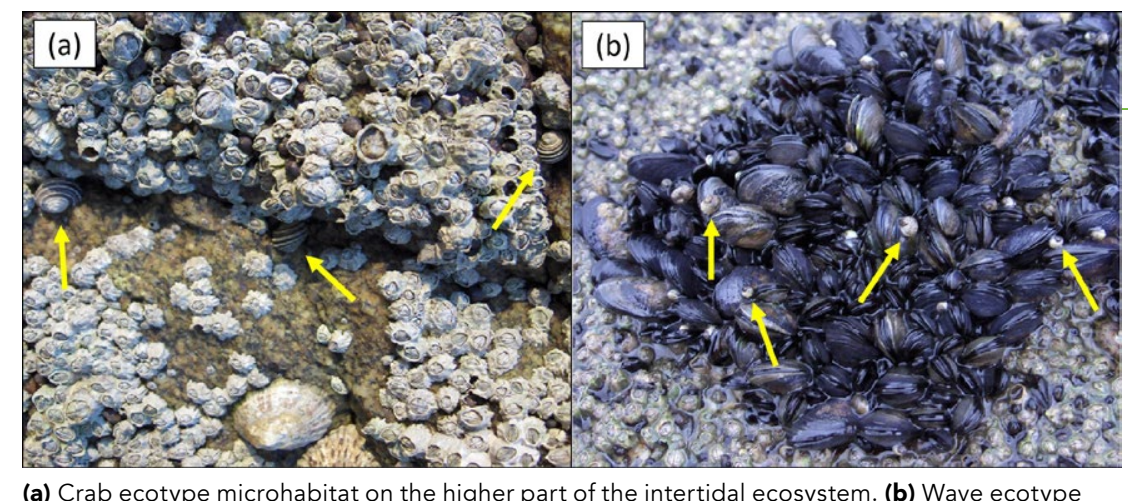

(a) Crab ecotype microhabitat on the higher part of the inter
microhabitat on the lower part of the intertidal ecosyste

change - in this example, the expected pattern is that the crab ecotype will change more than the wave ecotype

the wave ecotype should resemble the juvenile forms of the crab ecotype. This would mean that the shell aperture should (either in terms of shell size or proteomic differentiation), because the wave ecotyp is retaining its juvenile characteristics.

The direction of change indicates the type of change, for example, does it get bigger or smaller. For the wave ecotype, shell aperture should resemble the erly stape of the should cesemble the early as the hypothesis states that (due to paedomorphism) the adult forms of

werly size, but much faster in the crab ecotype. size, but much faster in the crab ecotype. Prof Rol't Ane analysis of the samples in Prof Rolán-Alvarez and colleagues' study aperting fit the expected dirizion to doesn't suggests that a different evolutionany process may be responsible for the divergence between the two ecotypes.

In L. saxatilis it is possible that paedomorphosis has resulted in the development of a separate ecotype adapted to life on lower levels of the shore.
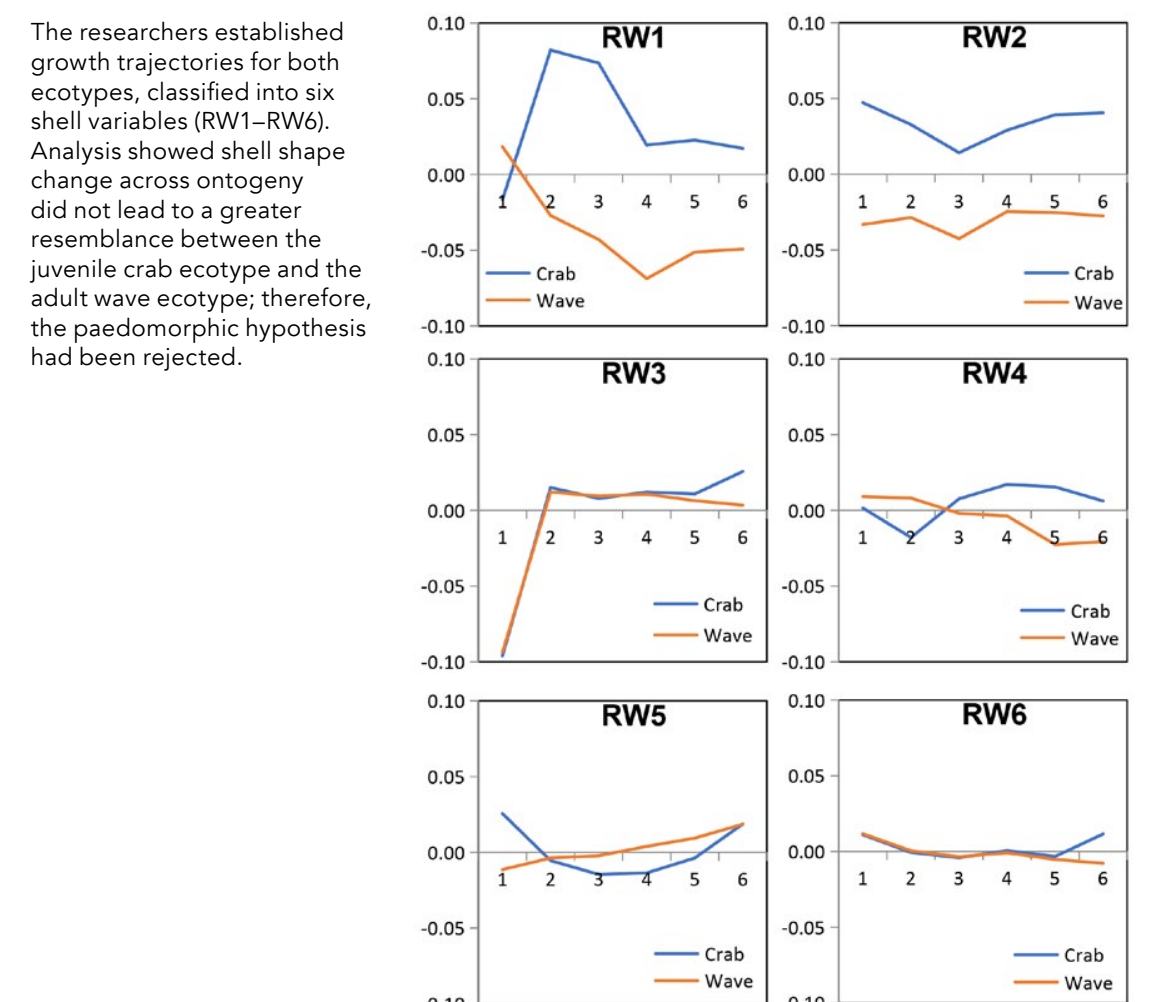
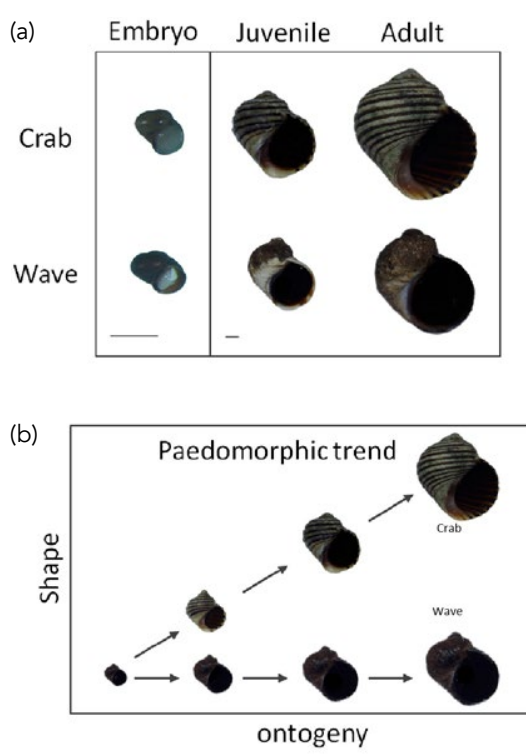

The paedomorphic hypothesis suggests the
dult forms of the wave ecotype adult forms of the wave ecotype resen
juvenile forms of the crab ecotype.

\section{EVO-DEVO} organism can have large impacts on the evolution of the species, but until recently developmental biology wasn't often a consideration when studying evolution. Evolutionary developmental biology, in development through processes such as paedomorphosis can influence the evolution of a species. In $L$. saxatilis it is possible that paedomorphosis has resulted in the development of a separate ecotype adapted to life on lower ( original morphology is nobut that he and it has been suggested that these two types could eventually diverge to become two species if further differentiation prevented interbreeding between the ecotypes. However, the evidence for paedomorphism in this species is not conclusive. Further investigation is required, and perhaps consideration of earlier development (the embryos in this study were fully formed) might provide a more definitive answer as to whether or not the L. saxatilis wave ecotype is an example of paedomorphism. Nevertheless, the analyses in these studies by Prof Rolan-Alvarez and his colleagues devo hypotheses at both mever devo hypotheses at both molecular and morphological levels, and to consider
both the pattern and direction of change.

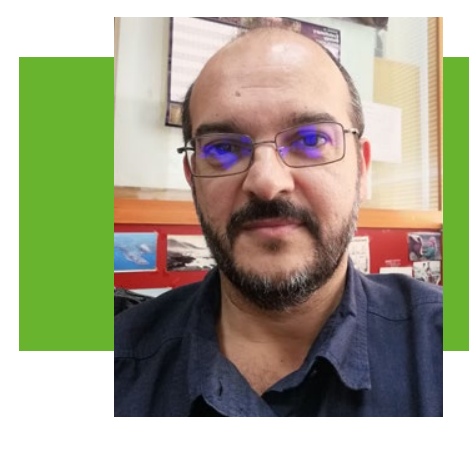

Behind the Research

Prof Emilio Rolán-Alvarez E: rolan@uvigo.es T: $+34986812578 \quad$ W: https://www.researchgate.net/profile/Emilio-Rolan-Alvarez
$\begin{array}{ll}\text { W: https://juangefaell.wordpress.com } & \text { W: } \text { http://genxb2.webs.uvigo.es }\end{array}$

\section{Research Objectives}

Prof Rolán-Alvarez tests whether the evolution of L. saxatilis ecotypes is consistent with the paedomorphic hypothesis.

\section{Detail}

\section{Address}

Lagoas-Marcosende, s/n, Facultad de Biologí

ersidad de Vigo

Prof Emilio Rolán-Alvarez has conducted research on evolutionary ecology and population genetics of marine snails, focusing on the causes of intraspecific variation mostly at very small scales at both phenotypic and molecular levels. These include the role of natural and sexual selection as well as the evolutionary mechanisms of speciation (evolution of reproductive isolation)

\section{Funding}

Xunta de Galicia (grant no. ED431C 2016-037), Fondos FEDER ('Unha maneira de facer Europa'), Ministerio de Economia Industria y Competitividad (grant no. CGL201675482-P). Galician Regional Government through the "Excellence in Research (NUGA) Program from the Regional Council of Culture, Education and Universities. The

\section{Collaborators}

The researchers would like to thank Mary Riádigos for administrative assistance, as well as DrManuela Truebano (Plymouth University) and two anonymous referees for

revising the last version of their manuscript.

Universida $_{\mathrm{de}}$ Vigo

\section{References}

Gefaell, J., Varela, N., and Rolán-Alvarez, E. (2020). Comparing shape along growth trajectories in two marine snail ecotypes of Littorina saxatilis. a test of evolution by paedomorphosis. Journal of Molluscan Studes, 86, 382-388. Available https://doi.org/10.1093/mollus/eyaa020

Diz, A.P., Páez de la Cadena, M., and Rolán-Alvarez, E. (2012). Proteomic evidence of a paedomorphic evolutionary process within a marine snail species: a strategy for adapting Biology 25(12) 2569-2581. Available at. https//doi org/10.1111/jeb.12001

\section{Personal Response}

\section{What inspired you to conduct this research?}

II We firmly believe that development is crucial to evolutionary mechanisms, especiallly natural selection, can provide knowledge about the survival of the fittest. we agree with developmental biologist Scott F. Gilbert that only the knowledge of development can help us answer questions about the arrival of the fittest. Given that for the relationship between evolution and development and given that we had previous evidence supporting it at the proteomic level, we wanted to test it at the most ecologically relevant trait in this species: shell shape.

How likely do you think it is that these two ecotypes III To some extent these two ecotypes are nearly separate species, as they have already developed choice, mating preferences, etc.) and have a reduced gene flow between them. Presently, several researchers are studying how much the gene flow is limited between them as, in fact, it seems to be more limited than previously thought. Of course formally they still of their genome, which make them an ideal system to understand how true species are formed. 